\title{
Análisis del patrón flexivo de los sustantivos relacionales en lengua mayangna
}

\author{
Ricard Viñas-de-Puig \\ (East Carolina University, EE.UU.) \\ Mayangna Yulbarangyang Balna'
}

\section{Introducción}

A pesar de que no existen muchas descripciones de las lenguas de la familia misumalpa en la bibliografía lingüística, son varios los autores que han escrito diferentes trabajos en los que se describen algunos de los fenómenos morfológicos y sintácticos de las distintas lenguas de esta familia (McCarthy y Prince 1993; Hale 1997; Norwood 1997; Benedicto y Hale 2000; KoontzGarboden 2007; Koontz-Garboden y Francez 2010; Eggleston et al 2011; entre otros). Con este artículo queremos complementar estos estudios analizando el comportamiento flexivo de los sustantivos relaciones (en adelante, SRs) en mayangna y comparándolo con el patrón de flexión de los demás elementos nominales de esta lengua. Según nuestra propuesta, la flexión de los sustantivos regulares sigue la estructura prosódica de la raíz (1), mientras que los SRs se flexionan siguiendo un patrón exclusivo para esta clase gramatical (2).
(1) a. walabis
b. walanibis
'niño'
b. Walanibis
'niño (3sg.)'
(2) a. $\mathrm{di} \mathrm{t}^{\mathrm{t}}$.
b. dinit
'debajo'
'debajo (3sg.)’

Teniendo en cuenta las divergencias de comportamiento flexivo de estos dos tipos de elementos nominales en mayangna, los objetivos que nos proponemos para este artículo son los siguientes: en primer lugar, presentar un resumen general de los diferentes patrones flexivos en mayangna, prestando especial atención al proceso de infijación en los sustantivos; y en segundo lugar, ofrecer un análisis actualizado del patrón flexivo de los SRs.

\subsection{Organización del artículo}

El presente artículo se divide en las partes siguientes. En la Sección 2, presentamos un resumen general de los patrones flexivos en mayangna, tanto en lo que aplica a sustantivos como a verbos y adjetivos. En la Sección 3 presentamos el uso y comportamiento de los SRs en mayangna, y hacemos énfasis en su forma de flexión, mientras que en la Sección 4 presentamos el análisis que da cuenta de su comportamiento flexivo. Por último, en la Sección 5 resumimos los puntos principales presentados y aportamos las conclusiones básicas a las que lleva este artículo.

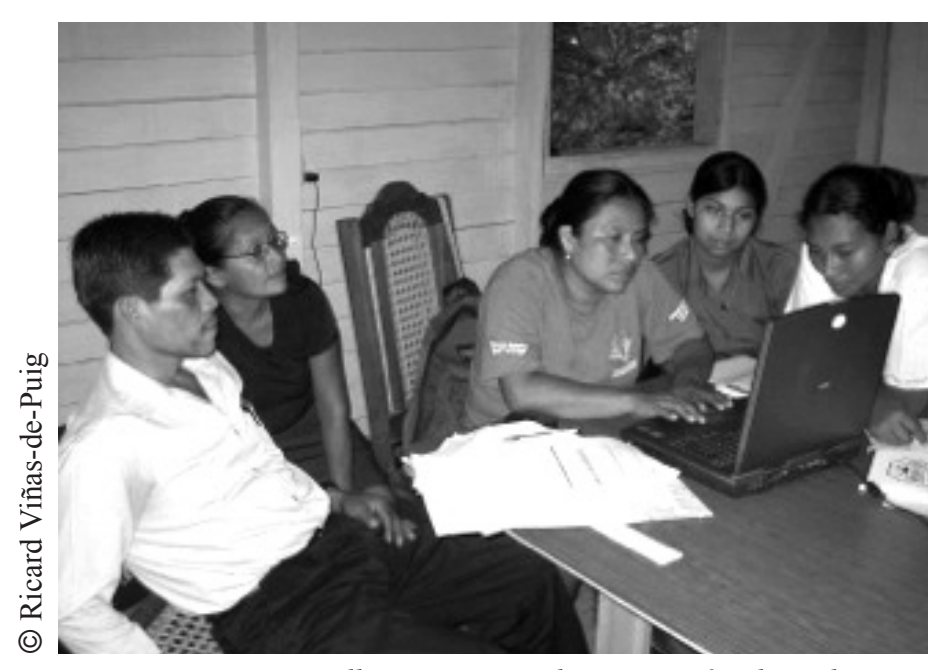

Equipo Mayangna Yulbarangyang Balna en sesión de trabajo en IPILC-Rosita

\footnotetext{
1 Equipo mayangna de lingüistas al que pertenecen los autores mayangnas de este artículo: Demetrio Antolín, Susana Budier, Modesta Dolores, Gloria Fenly, Tomasa Gómez y Elisa Salomón. Este equipo, asociado a IPILC-Rosita y coordinado por la Dra. Elena Benedicto, quedó establecido a finales de los años 1990. Desde su creación, el Mayangna Yulbarangyang Balna ha trabajado en la documentación de la lengua mayangna, en el estudio lingüístico de diferentes aspectos de la lengua, así como en la creación de materiales para promover el uso de la lengua en el ámbito escolar y comunitario.
} 


\section{Procesos de flexión en mayangna}

Como ya han indicado varios autores (ej. Benedicto y Hale 2000), en mayangna se observa la presencia de un sistema con base prosódica para obtener el patrón flexivo. Notamos, además, que los sustantivos se diferencian de los elementos predicativos (verbos y adjetivos) en lo que a la flexión se refiere. Según estos mismos autores, la base flexiva son los pies yámbicos, que pueden estar formados por una sílaba pesada o fuerte (con dos moras prosódicas) o por una sílaba inicial ligera (con una sola mora) seguida por otra sílaba (Benedicto y Hale 2000). Ésta es la base que se utiliza para determinar el lugar de inserción del afijo (en muchos casos, infijo) en los sustantivos o el lugar de reduplicación de los verbos y adjetivos. McCarthy y Prince (1993), en su análisis de la flexión de los posesivos en ulwa (dentro de los parámetros de la Teoría de la Optimidad), proponen una restricción de alineación relativa al lugar de colocación del afijo, como se observa en (3).

\section{(3) Alineación-Al-Pie (Ulwa)$$
\text { Alinear([POS } \left.]_{\mathrm{Af}}, \mathrm{Iz}, \mathrm{Pie}, \mathrm{D}\right)
$$

De acuerdo con esta restricción, en ulwa el afijo flexivo posesivo aparece a la derecha del primer pie prosódico. Siguiendo los análisis ya publicados en la bibliografía sobre el sistema flexivo del mayangna y el punto de afijación (Benedicto y Hale 2000; Norwood 1997; entre otros), argumentamos que en mayangna se puede aplicar la misma restricción de alineación, que queda modificada, como se muestra en (4).

$$
\begin{aligned}
& \text { Alineación-Al-PIE (Mayangna) } \\
& \text { Alinear([POS }]_{\mathrm{Af}} \text { Iz, Pie, D) }
\end{aligned}
$$

Así pues, según (4), en mayangna, al igual que en ulwa, el afijo posesivo en los sustantivos se inserta a la derecha del primer pie prosódico (en este caso, un pie yámbico).

\subsection{Flexión en el sistema nominal}

En mayangna, los sustantivos se pueden flexionar para indicar posesión o estado constructo. Según Benedicto y Hale (2000), este estado ocurre cuando el elemento nominal va precedido por un determinante o cuando el sustantivo, en su estado base, se interpreta como definido o determinado por el contexto. En los casos en los que se usa el estado constructo, los elementos nominales se flexionan usando el afijo de tercera persona del singular.
En los casos de flexión para indicar posesión, el afijo que se corresponde con la primera (-ki-), segunda (-ma-) o tercera (-ni-) persona se coloca después de la forma base de la raíz (4). Cabe recordar que la forma base es un yambo; en otras palabras, la base está formada por una sílaba pesada (una sílaba con una coda consonante (5) o una sílaba con una vocal larga (6)) o una sílaba ligera seguida por otra sílaba (ya sea ligera o pesada) (7). (Todos los ejemplos, salvo excepciones marcadas, se tomaron de la gramática de Norwood (1997).)

$$
\begin{aligned}
& \text { a. was 'agua' } \\
& \text { b. wasma 'agua (2sg.)' } \\
& \text { c. wasni 'agua (3sg.) }
\end{aligned}
$$
a. $\hat{\mathrm{u}}$
b. ûki
'casa'
c. ûni
'casa (1sg.)'
'casa (3sg.)'
a. kuring
'pipante'

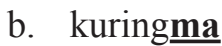
'pipante (2sg.)'

Este mismo patrón flexivo es el que se observa en los sustantivos formados por más de un pie prosódico, como se demuestra en los ejemplos (8) y (9) siguientes.

$$
\begin{array}{ll}
\text { a. pukta } & \text { 'noche' } \\
\text { b. pukmata } & \text { 'noche (2sg.)' } \\
\text { c. puknita 'noche (3sg.)' }
\end{array}
$$

$$
\begin{array}{ll}
\text { a. walabis 'niño' } & \\
\text { b. walakibis } & \text { 'niño (1sg.)' } \\
\text { c. walanibis } & \text { 'niño (3sg.)' }
\end{array}
$$

Es preciso destacar que en estos ejemplos, y de acuerdo con la restricción de alineación que presentamos en (4), el afijo se inserta justo a la derecha de la base y, en consecuencia, se convierte en un infijo.

\subsection{Flexión en los sistemas adjetival y verbal}

Tanto los adjetivos como los verbos comparten con los sustantivos la base que se utiliza para determinar la flexión. Sin embargo, hay diferencias más que sustanciales en el proceso de flexión de los adjetivos y los verbos cuando lo comparamos con el que observamos en los sustantivos.

La primera de las diferencias entre la flexión del sistema nominal y la de los adjetivos y verbos tiene que ver con el proceso morfológico que se da: si en los sustantivos 
notamos un proceso de afijación, en los verbos y adjetivos se observa un proceso de reduplicación parcial. Los adjetivos reduplican parte de la base para indicar pluralidad, mientras que los verbos reduplican únicamente en la tercera persona del plural (Norwood 1997; Benedicto y Hale 2000).

La segunda de las diferencias, que es precisamente la más relevante para este artículo, está relacionada con el lugar de flexión. Aunque, como ya mencionamos, tanto los sustantivos como los adjetivos y los verbos toman los pies yámbicos como base de flexión, en los verbos $\mathrm{y}$ adjetivos el elemento reduplicado se añade antes del elemento prominente del pie (Benedicto y Hale 2000). Este fenómeno se observa en los adjetivos siguientes, siguiendo los ejemplos de Norwood (1997).

$$
\begin{aligned}
& \text { a. salani 'gordo' salalani 'gordo (pl.)' } \\
& \text { b. parasni 'fuerte' pararasni 'fuerte (pl.)' } \\
& \text { c. sâni'negro' sasâni 'negro (pl.)' } \\
& \text { d. ingni 'ligero' ihingni 'ligero (pl.)' }
\end{aligned}
$$

Nótese que en los adjetivos anteriores, el elemento que reduplica no incluye la segunda mora: si la base contiene una coda consonántica, ésta no se incluye en la reduplicación (10b); si, por el contrario, la base contiene una vocal larga (bimoraica), sólo reduplica el valor corto de la vocal y, por lo tanto, el afijo reduplicante únicamente contiene una vocal corta $(10 \mathrm{c})$.

Además, el afijo reduplicante se inserta antes del elemento reduplicado. Se observa asimismo que si la sílaba prominente carece de arranque, el afijo reduplicante incluye una fricativa glotal en posición de coda, con el fin de romper un contacto de vocales idénticas (10d).

En el caso de los verbos, notamos un proceso idéntico, como se demuestra en los ejemplos (11) a (14) siguientes.
a. yulnin
'hablar
b. yulwi
'ella/él habla'
c. yuyulwi
'ellas/ellos hablan'
a. kasnin
'comer'
b. kaswi
'ella/él come'
c. kakaswi
'ellos comen'

(13)
a. dakânin 'oír'
b. dakâwi 'ella/él oye'
c. dakakâwi 'ellos oyen'

(14)
a. arknin
'roncar'
b. arkwi
'ella/él ronca'
c. aharkwi
'ellos roncan'

En mayangna, los verbos conjugados en tercera persona del plural presentan un proceso de reduplicación parcial idéntico al observado en los adjetivos en plural: el elemento prominente del pie prosódico se reduplica parcialmente ( $\sin$ que se reduplique la coda) y se inserta antes de la base. También en estos casos, si el elemento prominente de la base carece de arranque silábico, el afijo reduplicante incluye una $[\mathrm{h}]$ epentética, como se observa en el ejemplo (14c).

\section{SRs en mayangna: descripción y flexión}

$\mathrm{Al}$ igual que en muchas otras lenguas, en mayangna se observa la presencia de un grupo de elementos nominales, que conocemos como sustantivos relaciones (SRs). Como indican López y Sis, "(1)os sustantivos relacionales conforman un grupo de sustantivos que siempre van poseídos, nunca funcionan por sí solos. Su función es relacionar elementos dentro de la oración" (López y Sis 2004: 133). En mayangna, estos elementos nominales tienen como función expresar relaciones espaciales entre diferentes elementos discursivos (Norwood 1997: 102).

Si observamos más detenidamente el comportamiento morfológico de estos elementos en mayangna, podremos confirmar que los SRs siempre deben mostrar flexión. Este hecho los diferencia de los otros sustantivos, que pueden aparecer con o sin flexión (como se indica en la Sección 3.1.).

Pandinit kidi sanni duwi.

árbol debajo-3sg. Det. sombra ser-PRES-3sg.

'Hay una sombra debajo del árbol.'

Puesto que los SRs tienen naturaleza nominal, su flexión se realiza añadiendo un afijo a la raíz, que concuerda en persona con el elemento con el que se establece la relación espacial (como se observa en el ejemplo (15)). También, y como sería de esperar dada su naturaleza nominal, los SRs no reduplican, a diferencia de lo observado en verbos y adjetivos. 
A continuación, en el ejemplo (16) presentamos una lista (no exhaustiva) de los SRs en mayangna.
a. dinit 'debajo' b. minit 'encima'
c. dangnit 'detrás' d. tânit 'delante'
e. us $\underline{n i t}$ 'después, detrás' f. yaihnit 'al lado de'
g. muhnit 'enfrente' h. pasning 'dentro'
i. nining 'contra'

A pesar de las semejanzas que observamos entre los SRs y demás elementos nominales en mayangna, existen diferencias importantes entre la flexión de los SRs y los demás sustantivos. En primer lugar, el afijo flexivo no aparece después del elemento prominente de la base, sino que se añade antes de la coda consonántica. De hecho, en muchos casos, el infijo rompe un grupo consonántico en posición de coda silábica (16c, d, e, f, g, h).

Sin embargo, este fenómeno de eliminación de codas complejas no se da en todos los SRs: en algunos casos, el infjo aparece entre la vocal de la base y la coda consonántica (16a, b, i). Además, como ya notaron Benedicto y Hale (2000), en estos casos de infijación entre el núcleo silábico y la coda consonántica, la vocal del afijo provoca un efecto de copia vocálica (es decir, la vocal de la raíz se copia a partir de la vocal del afijo). Obsérvense los ejemplos (17) a (19).
a. dikit
'debajo (1sg.)'
b. damat
'debajo (2sg.)'
c. dinit
'debajo (3sg.)'
a. mikit
'encima (1sg.)'
b. mạmat
'encima (2sg.)'
c. mini
'encima (3sg.)'
a. niking 'contra (1 sg.)'
b. namang 'contra (2sg.)'
c. nining 'contra (3sg.)'

En estos ejemplos podemos observar como la vocal de la raíz cambia dependiendo del afijo que se inserta. Si el afijo contiene una [i] (como en los afijos de primera y tercera persona), la vocal de la raíz también será [i]; por el contrario, si la vocal del afijo es [a] (como en el afijo de segunda persona), la vocal de la raíz entonces será [a]. Cabe señalar que este fenómeno de copia vocálica no se da cuando el afijo rompe una coda compleja: en el ejemplo (16c) el afijo es el de marca de tercera persona del singular -ni-, pero la raíz mantiene su vocal original (dangnit 'detrás (3sg.)').

Si tenemos en cuenta estas diferencias entre la flexión de los SRs y los demás elementos nominales, se hace necesario proponer un análisis que pueda dar cuenta de estas divergencias. Éste es el objetivo de la Sección 4.

\section{Análisis del sistema flexivo de los SRs}

Como ya mencionamos, los SRs presentan un sistema flexivo diferente al de los demás sustantivos, especialmente en lo que se refiere al lugar de inserción del afijo y al proceso de copia vocálica observado en algunas raíces. En esta sección presentamos un análisis que da cuenta de los dos casos.

\subsection{Lugar de flexión}

Aunque ninguna de las fuentes consultadas hace un análisis profundo del asunto, la fonotáctica del mayangna parece indicar que existe una preferencia por la estructura silábica a $\mathrm{CV}(\mathrm{C})$, en los que se tienden a evitar los grupos consonánticos dentro de una misma sílaba. Un estudio de la obra de McLean (2005) demuestra que son contados los casos en los que encontramos grupos consonánticos dentro de una sílaba; además, en la mayoría de los casos, las palabras que los presentan son préstamos de otras lenguas. Esta idea de preferencia por una estructura silábica simple es un punto central del análisis que presentamos.

Según lo indicado en la sección anterior, en mayangna los SRs presentan un comportamiento flexivo que difiere del de los otros elementos nominales. Considérese de nuevo la flexión de un sustantivo común, repetida en el ejemplo (20).

$$
\begin{array}{ll}
\text { a. pukta } & \text { 'noche' } \\
\text { b. pukmata } & \text { 'noche (2sg.)' } \\
\text { c. puknita } & \text { 'noche (3sg.)' }
\end{array}
$$

Como podemos comprobar en el ejemplo anterior, en los sustantivos comunes el afijo flexivo aparece después del elemento prominente de la raíz (es decir, después del primer pie yámbico). El comportamiento de los SRs 
es diferente, como se puede observar en los ejemplos repetidos en (21).
a. dangnit 'detrás' b.usnit 'después, detrás'
c. yaihnit 'al lado de' d. muhnit 'enfrente'
e. pasning 'dentro'

En los SRs el afijo flexivo (de tercera persona) -ni- no aparece después del primer pie yámbico, sino que lo hace entre los diferentes elementos que formarían una coda compuesta (es decir, una coda con más de una consonante). Si el patrón flexivo de los SRs siguiera el de los demás sustantivos, esperaríamos obtener casos como los que mostramos en los ejemplos en (22).

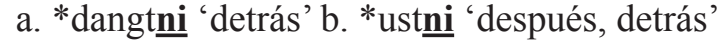
c. *yaihtni 'al lado de' d. *muhtni 'enfrente'
e. *pasngni 'dentro'

Sinembargo, los ejemplosen(22)nosonfonotácticamente posibles en mayangna. Como se afirma al principio de esta sección, en mayangna se observa una tendencia a mantener una estructura silábica de la forma $\mathrm{CV}(\mathrm{C})$, en la que no haya grupos consonánticos al inicio o final de sílaba. Por lo tanto, si los SRs de (21) siguieran el mismo patrón que los demás sustantivos, el resultado sería una estructura silábica con un grupo consonántico en la coda; es decir, tendríamos una estructura que se aleja de la preferida.

Así pues, podemos explicar el lugar de flexión si tomamos como válida una restricción que prohíba la presencia de grupos consonánticos. Prince y Smolensky (1993: 96), dentro del patrón general de la Teoría de la Optimidad, proponen la existencia tal restricción, observada en (23).

\section{(23) \\ *COMPLEX \\ No se puede asociar más de una $\mathrm{Co} \mathrm{V}$ a cualquier nodo de posición silábica.}

$\mathrm{Si}$ tomamos esta idea como válida, los grupos consonánticos dentro de una única sílaba (como los del ejemplo (22) anterior) supondrían una violación de esta restricción. Debemos notar, además, que esta restricción debe anteponerse a cualquier restricción de alienación relativa a la posición de los afijos con respecto al pie prosódico. Obtenemos así la jerarquía de restricciones que se observa en (24).

\section{(24) *COMPLeX $>$ Alineación-Al-Pie (Mayangna)}

Cabe notar que la jerarquía de restricciones anterior concuerda con la propuesta que presentan McCarthy y Prince (1993), según la que las restricciones fonológicas (como * COMPLEX) pueden tener mayor prioridad que las morfológicas (como AlineAción-Al-Pie (Mayangna)).

De acuerdo con este análisis y esta jerarquía de restricciones, podemos concluir que en mayangna los SRs forman una subclase de sustantivos, con una flexión basada en el patrón que presentamos en (25).

\section{(25) Patrón estructural para los SRs en MAYANGNA: $\mathrm{CV}(\mathrm{C}) \cdot[\mathrm{CV}]_{\text {afjo }} \mathrm{C}$}

Según este patrón, todos los SRs en mayangna están formados por una sílaba inicial, que puede tener (o no) una coda consonántica, seguida por el afijo flexivo y una consonante final.

Una vez presentado el análisis relativo al lugar de flexión de los SRs en mayangna, debemos presentar una solución a la variación observada en la copia de la vocal del afijo en la raíz. Este asunto se trata en el siguiente subapartado.

\subsection{Copia vocálica}

Ya indicamos que una de las diferencias observadas entre el comportamiento de los SRs y los demás sustantivos tiene que ver con el hecho de que algunos SRs copian la vocal del afijo en la raíz. Este fenómeno se ilustra en los ejemplos siguientes.
a. dikikt
b. damat
c. dinint
'debajo (1sg.)'
'debajo (2sg.)'
'debajo (3sg.)'
a. mikit
b. mamat
'encima (1sg.)'
c. minit
'encima (2sg.)'
'encima (3sg.)'
a. niking
'contra (1sg.)'
b. namang
c. nining
'contra (2sg.)'
'contra (3sg.)'

Como podemos ver en estos ejemplos, la vocal de la raíz es una copia de la vocal del afijo. Pero como ya dijimos, este proceso no se da en todos los SRs. 
Antes de presentar el análisis, podemos volver a echar un vistazo al patrón estructural que propusimos en el ejemplo (25) de la sección anterior. Según dicho patrón, todos los SRs están formados por una sílaba inicial que puede terminar con una coda consonántica (como en los ejemplos en (21)), o sin tal coda (como en los ejemplos de (26) a (28)). Si se da este segundo caso, se produce el fenómeno de copia de la vocal del afijo. Sin embargo, uno de los ejemplos hasta ahora mostrados parece indicar lo contrario. Obsérvese de nuevo el ejemplo (16d), repetido a continuación como ejemplo (29).

(29) tânit 'delante'

En este ejemplo debemos tener dos cuestiones en consideración. En primer lugar, cabe señalar que la primera sílaba no termina en una coda consonántica; en segundo lugar, a pesar de que la sílaba termine en vocal, este SR no presenta un caso de copia vocálica, en aparente contradicción con lo indicado anteriormente.

Para poder dar cuenta de esta aparente discrepancia, nos resulta necesario revisar el patrón estructural presentado en la sección anterior. Si analizamos de nuevo el ejemplo que presentamos en (29), podremos observar que la vocal de la raíz, - $-\hat{a}$, es una vocal larga, con un peso de dos moras. Si entendemos entonces que son las moras, y no las codas consonánticas, el elemento significativo en mayangna (y sabemos que las moras, y no sólo las consonantes, son relevantes para determinar lo que constituye un pie prosódico), entonces podremos presentar una revisión del patrón estructural presentado. Tenemos como resultado el patrón que presentamos en (30).

$$
\begin{aligned}
& \text { Patrón estructural para los SRs EN } \\
& \text { MAYANGna (ReVisado): } \mathrm{CV}(\mu) \cdot[\mathrm{CV}]_{\text {afijo }} \mathrm{C}
\end{aligned}
$$

De acuerdo con esta nueva versión del patrón estructural de los SRs, la primera sílaba puede estar formada por una o dos moras, y el afijo flexivo siempre irá seguido por una coda. La estructura silábica resultante queda ilustrada en el ejemplo (31).

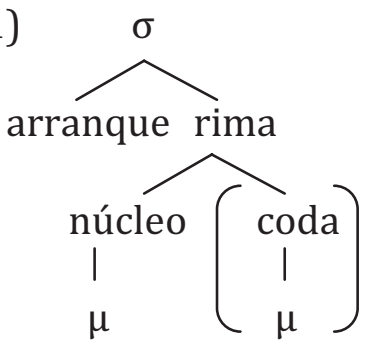

Ahora pues podemos tomar este patrón para presentar una explicación del fenómeno de copia vocálica observado sólo en algunos SRs. Recordemos que únicamente observamos copia vocálica si la primera sílaba de la raíz termina en vocal corta; es decir, si la primera sílaba sólo contiene una mora. En caso de que esto suceda, la raíz copiará la vocal del afijo. Dicho proceso queda ilustrado en el esquema que presentamos en (32), tomando el modelo de geometría de rasgos de Clements y Hume (1995).

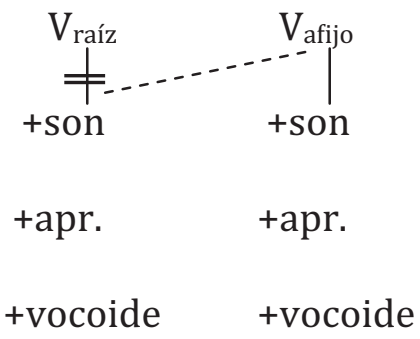

Sin embargo, y como ya se indicó en varias ocasiones, este proceso de copia vocálica no se da en otros SRs. La explicación a esta variación también está relacionado con el peso prosódico de la primera sílaba del SR: si la primera sílaba es bimoraica, la presencia de la segunda mora obstruye el proceso de copia vocálica, dando como resultado la posibilidad de tener dos vocales con valores dispares en las dos sílabas del SR. Este proceso queda ilustrado en el esquema siguiente (33).

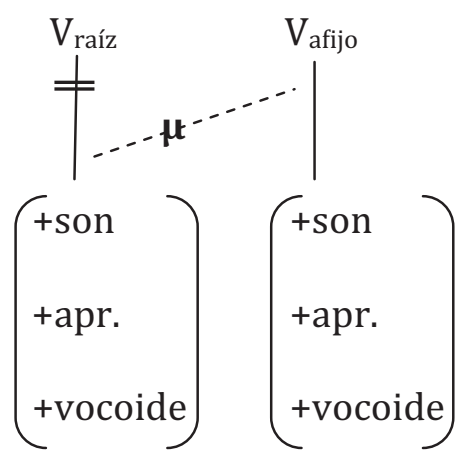

Mediante este análisis de patrones flexivos de los SRs en mayangna, podemos dar cuenta no sólo de la posición que ocupa el afijo (que impide que se formen sílabas con grupos consonánticos complejos), sino que también podemos explicar las restricciones relativas al fenómeno de copia de la vocal del afijo en la raíz. 


\section{Resumen y conclusiones}

En este artículo presentamos un análisis con el que cumplimos los objetivos que nos propusimos en la sección introductoria. Es decir, primero hicimos un resumen general de los patrones flexivos en mayangna, y llegamos a la conclusión de que la flexión sigue un patrón similar al que McCarthy y Prince (1993) propusieron para ulwa: los afijos flexivos en los sustantivos se añaden después del elemento prominente del pie prosódico (pie yámbico), siguiendo la restricción Alineación-Al-Pie (Mayangna).

En segundo lugar, hicimos hincapié en la flexión de los SRs de mayangna. Después de notar que estos elementos presentan un sistema flexivo que difiere del de los demás sustantivos (en lo que se refiere al lugar de inclusión del afijo y a la estructura silábica de la raíz), presentamos un análisis según el cual los SRs forman una sublcase dentro de los elementos nominales. La flexión de estos sustantivos sigue el patrón $\mathrm{CV}(\mu)[\mathrm{CV}]_{\text {afijo }} \mathrm{C}$, que es el resultado de la aplicación de una restricción *CompLEX que impide que se formen grupos consonánticos dentro de una misma sílaba. Además, señalamos que dicha restricción se antepone a la restricción que hace referencia al lugar de fijación del afijo, ya que un orden diferente de esas dos restricciones da como resultado formas agramaticales.

Por último, proponemos que este patrón de estructura silábica también sirve de base para el fenómeno de copia vocálica observado en un pequeño grupo de SRs. Según este patrón, la ausencia de una segunda mora en la sílaba inicial de la raíz favorece que la vocal del afijo se pueda copiar como núcleo de la raíz; sin embargo, si la sílaba inicial es bimoraica (con una consonante en posición de coda o con una vocal larga), la segunda mora impedirá que se produzca copia vocálica, abriendo así la posibilidad para que la vocal de la raíz y la del afijo puedan ser diferentes. 


\section{Bibliografía}

Benedicto, Elena, y Ken Hale. 2000. "Mayangna, a Sumu language: Its variants and its status within Misumalpan”. University of Massachusetts Occasional Papers 20: 75-106.

Clements, George N., y Elizabeth Hume. 1995. "The internal organization of speech sounds". En: Goldsmith, John, eds. Handbook of Phonological Theory. Oxford: Basil Blackwell. 245-306.

Eggleston, Alyson; Elena Benedicto; and Mayangna Yulbarangyang Balna. 2011. "Spatial frames of reference in Sumu Mayangna". Language Sciences 33 (6): 1047-1072.

Hale, Ken. 1997. “The Misumalpan causative construction”. En: Bybee, Joan, John Haiman, y Sandra A. Thompson, eds. Essays on language function and language type: Dedicated to T. Givon. Amsterdam: John Benjamins. 199-216.

Koontz-Garboden, Andrew. 2007. States, changes of state, and the Monotonicity Hypothesis. PhD dissertation. Stanford University.

Koontz-Garboden, Andrew y Itamar Francez. 2010. “Possessed properties in ulwa”. Natural Language Semantics 18 (2): 197-240

López, Candelaria, y María Juliana Sis. 2004. Gramática Pedagógica K’iche’. Guatemala: Universidad Rafael Landívar, Instituto de Lingüística y Educación.

McLean, Melba, y Miguel Urbina. 2005. Diccionario trilingüe. Miskitu, Sumu/Mayangna, Español. Managua, Nicaragua: CIDCA-UCA.

Norwood, Susan. 1997. Gramática de la Lengua Sumu. Managua, Nicaragua: CIDCA.

Prince, Alan, y Paul Smolensky. 1993. “Optimality Theory: Constraint Interaction in Generative Grammar”. RuCCS-TR-2. ROA-537. 